\title{
CT Diagnosis of Fahr disease, a case report
}

\section{Antonio Gligorievski \\ University Clinic for Surgical Diseases "St. Naum Ohridski" Skopje, Macedonia}

\section{Introduction}

Fahr's disease is a rare inherited or sporadic neurological disorder with a prevalence of $<1 / 1,000,000$, with a higher incidence reported among males and a typical age of onset in the 4th-6th 3rd-5th decade of life [1]. Fahr's disease, or idiopathic striopallidodentate calcinosis, or idiopathic basal ganglia calcification is a rare clinical entity characterized by bilateral and symmetrical intracerebral calcifications of the basal ganglia, thalamus, dentate nucleus and centrum semiovale in the absence of any metabolic abnormality including hypoparathyroidism [2,3]. Within the basal ganglia, the globus pallidus is the most frequent site of calcification, but deposits may be present in the putamen, caudate nucleus, and internal capsule, dentate nucleus of the cerebellum, thalamus, and the periventricular white matter [4,5]. Sporadic and family cases have been reported $[4,5]$. It is usually inherited by an autosomal dominant pattern, although sporadic and autosomal recessive cases had also been reported. In 1930, the calcifications of the basal ganglia named "Fahr's disease", after the German neuropathologist Karl Theodor Fahr [6]. The disease was first radiologically defined it by Fritzcher in 1935 [7]. Fahr's disease should be differentiated from Fahr's syndrome. The Fahr's disease is used for primary basal ganglia calcification, and the Fahr syndrome is used for basal ganglia calcifications associated with low serum levels of calcium and phosphorus, which usually occur in hypoparathyroidism [8-10]. Both entities are defined by bilateral intracranial calcification with neuropsychiatric symptoms and extrapyramidal disorder [11].

The most common neurological signs are a headache, vertigo, syncope, movement disorders, seizures, paresis, spasticity, speech disorders, parkinsonism, chorea, and orthostatic hypotension [4,5] but it is also known that the disease may be completely asymptomatic [12].

Computed tomography is the preferable method of localizing and assessing the extent of cerebral calcification. The most frequently affected area is the lenticular nucleus, especially the globus pallidus. Calcifications in the putamen, thalami, caudate, and dentate nuclei are also common. Calcification seems to be progressive and gradual. However, because of the frequency of asymptomatic patients, the incidental diagnosis in patients undergoing brain CT scan for other reasons is possible.

\section{Presentation of case (Case report)}

Here in, we present a case of a 56-years-old male with a 6 months history of seizures and headache came for noncontrast computed tomography of the brain. Clinical examination revealed normal orientation to time, place and person with a normal level of consciousness. The neurological and physical examination was unremarkable. Laboratory examinations including blood levels of glucose, iron, calcium, ferritin, and parathormone were within normal limits.
CT of the brain revealed bilateral and symmetric, extensive, irregular, amorphous, calcifications involving the basal ganglia (caudate nucleus, globus pallidus, lentiform nucleus, and putamen), thalamus and dentate nucleus (Figures 1-4). Axial Noncontrast CT images of the brain in the level of posterior fossa show bilateral, dense and extensive calcifications involving the dentate nuclei and white matter of cerebellum with preserved cerebellar hemispheres. Note the "sun ray" calcification around the bilateral dentate nuclei (Figure 1). Axial Noncontrast CT images of the brain in the level of basal ganglia and lateral ventricles show bilateral, dense and extensive calcifications in the basal ganglia (caudate nucleus, globus pallidus, lentiform nucleus, and putamen). It is evident that the brain parenchyma does not show focal pathological changes. Calcifications are also seen in the choroid plexus of the ventricular system (Figure 2).

Based on the clinico-radiological and biochemical findings, the provisional diagnosis of idiopathic striopallidodentate calcinosis or Fahr's disease was strongly suggested.

\section{Discussion}

Fahr's disease is characterized by clinical heterogeneity and can be asymptomatic or associated with neuropsychiatric manifestations $[4,5,12,13]$. Fahr's disease is more frequent among middle-aged patients.

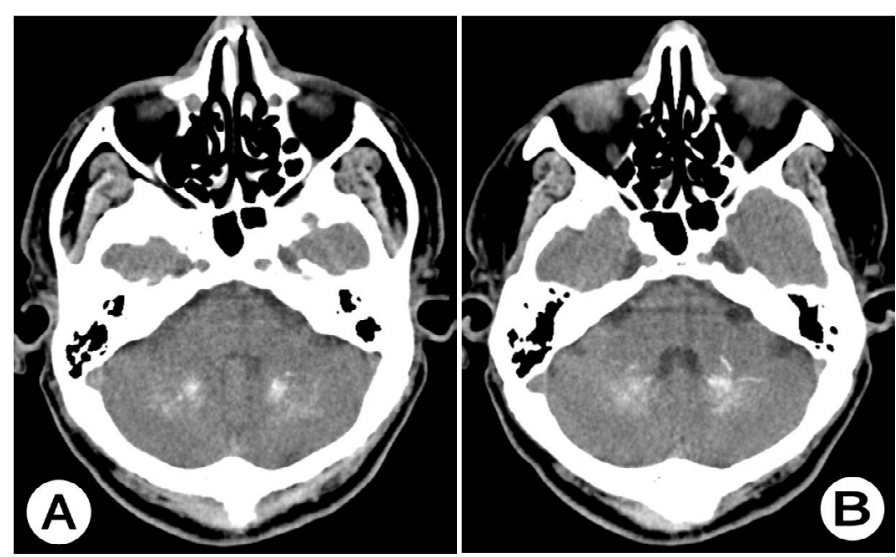

Figure 1. Noncontrast CT of the brain in the level of posterior fossa (A) and (B): Axial CT images show bilateral, dense and extensive calcifications involving the dentate nuclei and white matter of cerebellum with preserved cerebellar hemispheres in 56 years old man. Note the "sun ray" calcification around the bilateral dentate nuclei.

*Correspondence to: Antonio Gligorievski, University Clinic for Surgical Diseases "St. Naum Ohridski", ul. Vasil Gjorgov br. 28/25, 1000, Skopje, Macedonia, E-mail: atglmed@gmail.com

Received: January 23, 2018; Accepted: February 23, 2018; Published: February 26,2018 

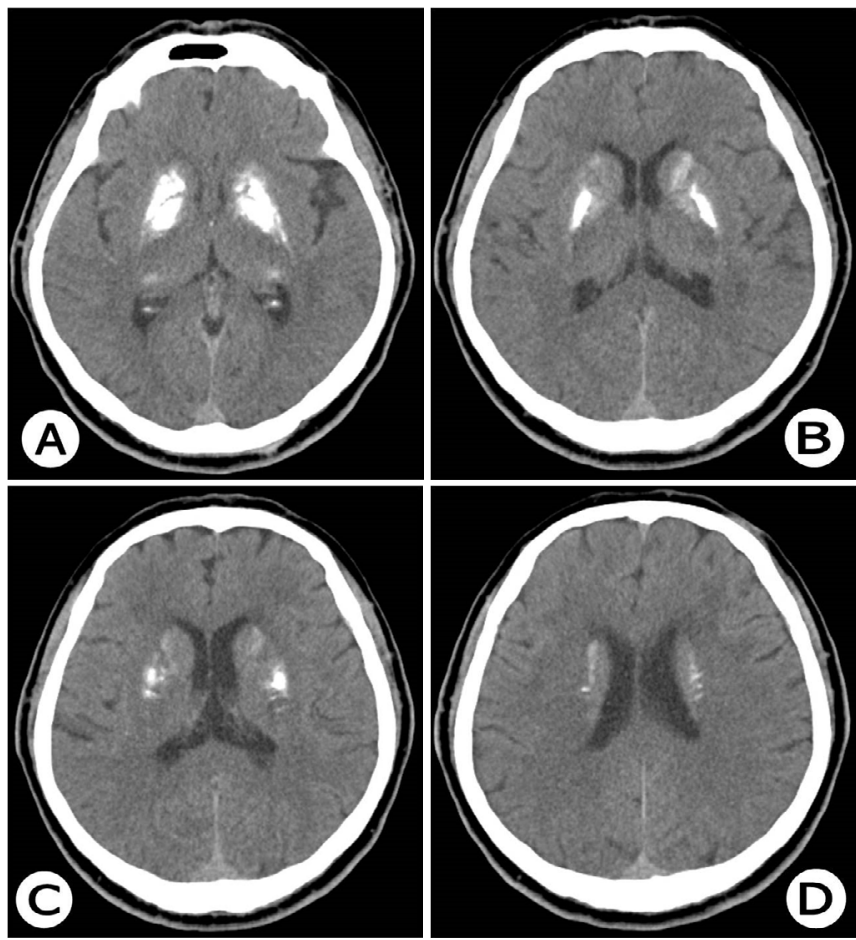

Figure 2. Noncontrast $\mathrm{CT}$ of the brain in the level of basal ganglia and lateral ventricles (A) and (B): Axial CT images clearly show bilateral, dense and extensive calcifications in the basal ganglia (caudate nucleus, globus pallidus, lentiform nucleus, and putamen) in 56 years old man. It is noticeable that the brain parenchyma does not show focal pathological changes. Calcifications are also seen in the choroid plexus of the ventricular system.

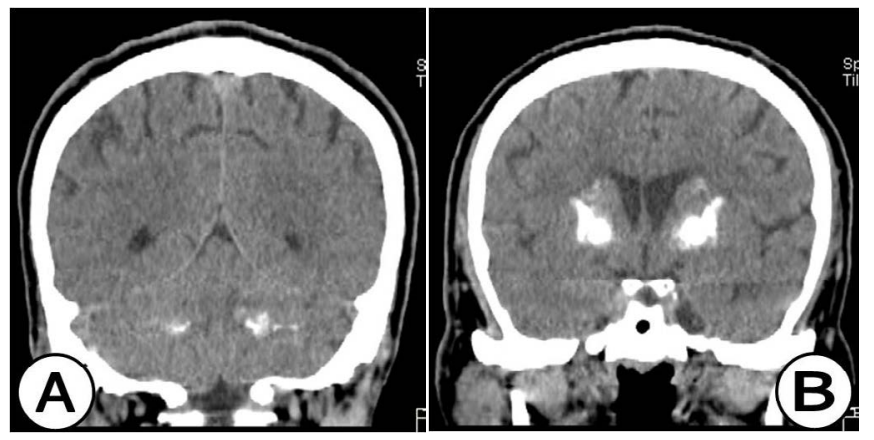

Figure 3. Coronal reformatted noncontrast CT scan images of the brain. (A) In the level of posterior fosse CT image clearly show bilateral, dense and extensive calcifications involving the dentate nuclei and white matter of cerebellum. (B) In the level of the frontal horns of the lateral ventricles, CT image clearly shows bilateral, dense and extensive calcifications in the basal ganglia (caudate nucleus, globus pallidus, lentiform nucleus, and putamen) in 56 years old man.

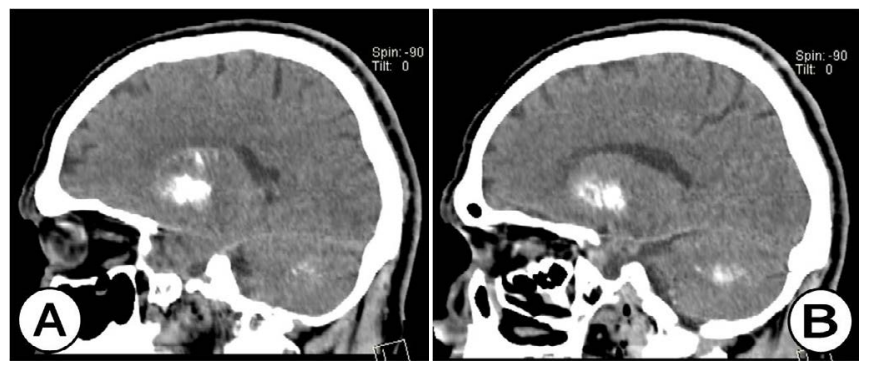

Figure 4. Sagittal reformatted noncontrast $\mathrm{CT}$ scan images of the brain (A) and (B): CT image clearly show dense and extensive calcifications involving the dentate nuclei and white matter of cerebellum. Also clearly show dense and extensive calcifications in the basal ganglia (caudate nucleus, globus pallidus, lentiform nucleus, and putamen) in 56 years old man.
The true prevalence of Fahr's disease is unknown, but an incidence of basal ganglia calcifications ranging from $0.24 \%$ to $2 \%$ has been reported in consecutive radiologic studies, showing an evident relationship with increasing age [13,14,]. Tedrus et al. [13] reported an incidence of $0.68 \%$ among 3.662 cranial CT scans analyzed. It should be emphasized that there are other conditions like hypothyroidism, systemic scleroderma, systemic lupus erythematosus, toxoplasmosis, neurocysticercosis, neurobrucellosis, tuberous sclerosis, neonatal anoxia, idiopathic hemochromatosis, heavy metal and carbon monoxide intoxication, and radiotherapy can also produce intracerebral calcifications [4,13-15]. These diseases cause bilateral and non-symmetric cerebral calcifications mainly located in the basal ganglia and cerebellum $[4,13]$.

Fahr's disease should be diagnosed based on clinical aspects, neuroimaging findings and the exclusion of other primary causes. It may occur in a sporadic or familial manner [4,5]. Geschwind et al. [16], in a genetic study, described a dominant autosomal inheritance of the hereditary form of Fahr's disease and suggested that the disease is caused by mutations in genes located on the long arm of chromosome 14 .

The main neurological manifestations of FD include motor disorders such as parkinsonism, dystonia, tics, speech alterations, epilepsia, and motor deficits, which are probably due to the involvement of the frontostriatal motor system $[4,5,17]$.

The most common radiologic feature of Fahr's disease is the presence of small bilateral intracranial calcifications which are usually restricted to the globus pallidus, but may also affect the putamen, caudate nucleus, thalamus, dentate nucleus and white matter of the cerebral hemispheres [5,18-20]. Lester et al. [18] reported an atypical case of Fahr's disease which developed stroke-like symptoms and progressed to extrapyramidal syndrome. Cranial CT demonstrated extensive bilateral and symmetric intracranial calcifications located at usual sites such as the basal ganglia and cerebellum and anusual sites such as the temporal and frontal periventricular white matter and semioval centers. Intracranial calcifications can be detected incidentally in up to $0.3-1.2 \%$ of CT examinations of the brain. 3 CT scanning is an easy test with maximum sensitivity and allows the easy diagnosis of Fahr's disease [21,22]. Radiologists may detect bilateral abnormalities of the basal ganglia and thalamus in different acute and chronic clinical situations. The neuroimaging diagnosis is also influenced by detection of abnormalities involving other parts of the brain, especially the cerebral cortex, brainstem, and white matter. Important alternatives in the radiologic differential diagnosis for Fahr's disease include hypoparathyroidism or pseudohypoparathyroidism (end-organ resistance to parathyroid hormone), which can be confirmed with measurements of serum calcium, phosphorus, and parathyroid hormone levels. Pseudohypoparathyroidism, in which there is no abnormality of calcium metabolism in asymptomatic patients, is another possible diagnosis in patients with widespread cerebral calcification [23].

Some of the intracranial structures calcify and considered physiological with aging, are pineal gland $60 \%$, habenular commissure $30 \%$, choroids plexus $10 \%$, dura mater $7 \%$, petroclenoid, interclenoid ligaments $12 \%$, pituitary gland and carotid arteries [24]. There are various causes of pathological calcifications in brain parenchyma, they may be infective, traumatic, metabolic and congenital conditions [21].

Intracranial calcifications may sometimes be observed on plain radiographs and frequently revealed by CT in normal older people. CT is the best modality to evaluate intracranial calcifications. 
Regarding this case the patient's age, presenting symptoms, location and volume of calcification are so extensive is suggestive to diagnose the Fahr's disease according to criteria described in the various literature.

\section{Conflict of interest}

Dr. Antonio Gligorievski declare that there is no conflict of interest.

\section{Funding}

In this case report there are no sponsors.

\section{Ethical approval}

Ethical approval was not necessary in this case report.

\section{Consent}

Written informed consent was obtained from the patient for publication of this case report and accompanying images. A copy of the written informed consent is available for review by the Editor in Chief of this journal on request.

\section{References}

1. Manyam BV, Walters AS, Narla KR (2001) Bilateral striopallido dentate calcinosis: clinical characteristics of patients seen in a registry. Mov Disord 16: 258-264. [Crossref]

2. Mittal A, Agrawal BK, Mittal A, Gupta P, Jain A (2010) Fahr's syndrome: a rare case of idiopathic basal ganglia calcification. JIACM 11:239-241.

3. Khammassi N, Chrifi J, Mohsen D, Abdelhedi H, Tougourti MN, et al. (2010) [Fahr's syndrome: two case report]. Rev Neurol (Paris) 166: 446-450. [Crossref]

4. Chevalier D, Marie I, Tillon J, Lévesque H (2005) A cause of cerebral calcifications not to be ignored: Fahr syndrome. Rev Med Interne 26: 668-677. [Crossref]

5. Manyam BV (2005) What is and what is not 'Fahr's disease'. Parkinsonism Relat Disord 11: 73-80. [Crossref]

6. Fahr T (1930) Idiopathische Verkalkung der Hirngefässe. Zentralb $\neg$ latt für allgemeine Pathologie und pathologische Anatomie 50: 129-133.

7. Scotti G, Scialfa G, Tampieri D, Landoni L (1985) MR imaging in Fahr disease. $J$ Comput Assist Tomogr 9: 790-792. [Crossref]

8. Ashtari F, Fatehi F (2010) Fahr's disease: variable presentations in a family. Neurol Sci 31: 665-667. [Crossref]
9. Watts RL, Koller WC (2004) Movement disorders: neurologic principles \& practice, (2ndedn), McGraw-Hill Professional, 2004, 550.

10. Malamud N, Hirano A (1974) Atlas of neuropathology, (2ndedn), University of California Press, $410-411$.

11. Murphy MJ (1979) Clinical correlations of CT scan-detected calcifications of the basal ganglia. Ann Neurol 6: 507-511. [Crossref]

12. Modrego PJ, Mojonero J, Serrano M, Fayed N (2005) Fahr's syndrome presenting with pure and progressive presenile dementia. Neurol Sci 26: 367-9. [Crossref]

13. Tedrus GMAS, Fonseca LC, Nogueira EJ (2006) Basal ganglia calcification on computed tomography: clinical characteristics in 25 patients. Arq Neuropsiquiatr 64: 645-649.

14. Lam JS, Fong SY, Yiu GC, Wing YK (2007) Fahr's disease: a differential diagnosis of frontal lobe syndrome. Hong Kong Med J 13: 75-77. [Crossref]

15. Faria AV, Pereira IC, Nanni L (2004) Computerized tomography findings in Fahr's syndrome. Arq Neuropsiquiatr 62: 789-792. [Crossref]

16. Geschwind DH, Loginov M, Stern JM (1999) Identification of a locus on chromosome 14q for idiopathic basal ganglia calcification (Fahr disease). Am J Hum Genet 65: 764772. [Crossref]

17. Weisman DC, Yaari R, Hansen LA, Thal LJ (2007) Density of the brain, decline of the mind: an atypical case of Fahr disease. Arch Neurol 64: 756-757. [Crossref]

18. Lester J, Zúñiga C, Díaz S, Rugilo C, Micheli F (2006) Diffuse intracranial calcinosis: Fahr disease. Arch Neurol 63: 1806-1807. [Crossref]

19. Schmidt U, Mursch K, Halatsch ME (2005) Symmetrical intracerebral and intracerebellar calcification ("Fahr's disease"). Funct Neurol 20: 15. [Crossref]

20. Shouyama M, Kitabata Y, Kaku T, Shinosaki K (2005) Evaluation of regional cerebral blood flow in fahr disease with schizophrenia-like psychosis: a case report. AJNR Am J Neuroradiol 26: 2527-2529. [Crossref]

21. Fenelon G, Gray F, Paillard F, Thibierge M, Mahieux F, et al. (1993) A prospective study of patients with CT detected pallidal calcifications. J Neurol Neurosurg Psychiatry 56: 622-625. [Crossref]

22. Shigeyuki O (2002) Imaging of bilateral striopallidodentate calcinosis. Clin Nucl Med 27: 721-724. [Crossref]

23. Preusser M, Kitzwoegerer M, Budka H, Brugger S (2007) Bilateral striopallidodentate calcification (Fahr's syndrome) and multiple system atrophy in a patient with longstanding hypoparathyroidism. Neuropathology 27: 453-456. [Crossref]

24. Lane BA, Moseley IS, Theron J (1999) The skull and brain. Methods of examination: diagnostic approach. In: Grainger RG et al. (1992) Diagnostic radiology (Vol 3), Churchil Livingstone; 90: 1909.

Copyright: (C2018 Gligorievski A. This is an open-access article distributed under the terms of the Creative Commons Attribution License, which permits unrestricted use, distribution, and reproduction in any medium, provided the original author and source are credited. 